\title{
Fighting Fire with Fire: Optimality of Value Destruction to Mitigate Short-Termism
}

\begin{abstract}
Adrian Aycan Corum*
\end{abstract}
May 9, 2021

Preliminary and incomplete

\begin{abstract}
I study a model of short-termism where a firm's value is affected by the actions of an agent, who can represent the manager or the board, as well as an entrepreneur, venture capital, private equity, or activist shareholder. The agent either has a project with positive NPV and can further increase the NPV by exerting effort, or has a project that destroys value. The agent has a stake in the company and can liquidate it before the NPV of his actions is realized by the market. This ability to exit creates short-term incentives for the agent to not exert effort, as well as opportunities for him to profit even if he is destroying value. I find that replacing a fraction of agents that have positive NPV projects with value-destroying agents can increase average firm value, because it motivates the valuecreating agents to work harder and this effect can dominate the added value destruction. Moreover, this result also holds under endogenous entry of agents: Reducing the entry or operating costs for the agents can increase average firm value and gross value destruction simultaneously, even though the fraction of agents with positive NPV projects decreases. Therefore, regulations that aim mitigating short-termism by curbing value destruction can actually yield opposite results and reduce average firm value instead.

Keywords: Myopia, Short-Termism, Incentives, Corporate Investment, Entrepreneurship, IPO, Venture Capital, Private Equity, Shareholder Activism, Blockholder.

JeL Classification: C70, D74, D83, G23, G34
\end{abstract}

*Johnson Graduate School of Management, Cornell University, corum@cornell.edu. I thank Murillo Campello, Vyacheslav Fos, Doron Levit, and Nadya Malenko for helpful comments. 


\section{Introduction}

Short-termism is a major topic that draws the attention of policymakers, academics, and practitioners all over the world. In a nutshell, short-termism can be summarized as the problem of sacrificing long-term value for short-term gains. A very common reason why this problem arises is because the market often cannot accurately assess the NPV of the actions undertaken by firms. Due to this friction, if an agent who can affect the firm value also has opportunities to liquidate his stake in the firm before the NPV is realized, the agent may profit from deliberately undertaking actions that seem beneficial for the firm but are actually detrimental to the firm value. When this is the case, it might be tempting to argue that such value destruction should be prevented. However, from a rational standpoint, the reason why such an agent can profit from undertaking a value-destroying action in the first place is that he can "pool" with other agents who create value. Otherwise, the stock price would go down and the former agent would make a loss instead. Therefore, the flip side of the coin is the incentives of the latter kind of agents regarding how much effort to exert and how much value to create as a result. In this paper, I show that a higher number of value-destroying agents can result in more effort by the other agents, and moreover, the overall impact on the average firm value can be positive as a result.

In particular, I develop a model where a firm's value is affected by the actions of an agent, who can represent the manager or the board, as well as an entrepreneur, venture capital, private equity, or activist shareholder. The agent has either a "good" or "bad" project, which is the private information of the agent: In the former case the agent's project is positive NPV and the agent can further increase it by exerting effort. In the latter, the agent's project has negative NPV. The main friction in the model is that, as alluded to above, it takes time for the NPV of the project to be realized, and the agent can liquidate his stake in the company (i.e., "exit") before the NPV is realized. With some probability, the agent has to sell his stake 
(e.g., due to a liquidity shock or an attractive outside investment opportunity), making exit on the equilibrium path for any project that the agent might have.

The exit opportunity of the agent creates short-term incentives for him not to exert effort. To see this, consider the case where there are no agents with negative NPV projects. Then, for any cost of effort, the agent with good project does not exert effort with some probability. This is because if he did, then the stock price would incorporate this expectation, and therefore the agent would have incentives to not exert effort and would sell his stake instead. In other words, in equilibrium, the first-best is not achieved due to short-termism.

The key result of the paper is that if the cost of effort is not prohibitively high (that is, the agent with positive NPV project exerts effort with some probability), average firm value increases as the fraction of agents that destroy value increases up to a certain limit. This is because a value-destroying agent always sells his stake, and therefore as the fraction of these agents increase, the stock price reacts more negatively upon the agent's sale decision. As a result, the incentives of the agents with positive NPV projects to keep their stake until the NPV is realized increases. In turn, these agents internalize the effect of their actions on the actual firm value more, and therefore their incentives to exert effort to further increase the value of the firm also get stronger.

Importantly, I show that this positive effect described dominates the greater value destruction induced by the higher fraction of agents with negative NPV projects. The intuition is as follows. As the fraction of value-destroying agents increases, the agents with positive NPV projects increase their effort to a certain point. In particular, their higher effort increases the average stock price upon their exit to the point where they become once again indifferent between exerting effort (and keeping their stake) vs. not exerting effort (and selling their stake). The critical observation is that the impact of higher effort of the agent has more positive impact on the unconditional average value of the firm compared to the average stock price conditional on the exit of the agent. This is because upon the exit of the agent, the market assigns a lower 
probability that the agent has exerted effort, since such an agent does not exit unless he has to. Therefore, as the agent with positive NPV project exerts more effort and the agent's exit price reaches its original level, this higher effort results in even a larger increase in the unconditional firm value and pushes it up beyond its old level. This is how the average firm value increases as the fraction of value-destroying agents increases, and it holds all the way until this fraction is sufficiently high so that the agents with positive NPV projects always exert effort and keep their stake (if possible). Overall, to put it differently, one kind of short-termism (i.e., not exerting sufficient effort while still creating value) can be alleviated with what can be seen as even more severe short-termism (i.e., outright value destruction).

While the arguments above abstract from career concerns, as I discuss in the paper, the results would not get weaker (and instead sometimes get stronger) if career concerns were incorporated in my model as well. In contrast, the main result (i.e., that a higher ratio of value-destroying agents can increase average firm value) does not necessarily continue to hold in an alternative model where career concerns replace short-termism (which stems from the opportunity for the agent to sell the stake before the NPV is realized). An important reason for this, among others, is that the modeling of the agent in this paper captures more than the skillfulness of the agent. In particular, if the quality of the agents' projects now and in the future are independent from each other, then incorporating career concerns would have no impact on the agents' incentives, since the market wouldn't make any inference from the NPV of the agent's current project about the NPV of his future projects (for simplicity, my model consists of a single stage where an agent implements a project only once, but it could be easily extended to a repeated game setting where the agent can implement different projects over time, and the results would continue to hold).

However, the results of my model continue to hold even if the agent has a level of "skill" (i.e., there is a positive correlation between the quality of an agent's projects over time). Therefore, an implication of the model is that an agent that has proven his skill over time might start 
creating less value compared to an agent that hasn't proved his skill yet, even if there are no career concerns involved.

Moreover, regardless of whether there is any element of skill or career concerns, the model also has interesting implications for the case when there is a principal in charge of choosing the agent. First, if the principal is facing two different agents' proposals at the same time (for example, shareholders of a firm voting for the board of directors vs. an activist in a proxy fight), it might be optimal for the principal to follow the proposal of the agent who is more likely to destroy value. Second, whenever there is a principal that selects the agent (e.g., the board choosing a CEO), it might be optimal for the principal to be not too expert in identifying whether the agent has positive vs. negative NPV projects (or alternatively, whether the agent is skilled vs. unskilled, if there is a skill component). This is because if the principal can perfectly distinguish between these agents, then she strictly prefers the agent that creates value. However, as described above, such an agent might create less value compared to an agent that destroys value with some probability.

Next, I endogenize the fraction of agents that implement value-destroying projects, since doing so enables studying effects of regulations that might impact this fraction and the average quality of projects as a result. In particular, I assume that agents have some entry or operating costs, which represent the expenses the agent needs to incur to start or run his business. Alternatively, this cost can also represent the agent's cost of implementing his project (which, in this case, would be in addition to the cost that the agent with good project incurs to exert effort). In addition, I also add the flexibility to the model to account for the price paid by the agent to obtain his stake. A value-destroying agent can make profit because he can sell his stake before the NPV is realized. However, as more agents implement negative NPV projects, the market incorporates this expectation into the stock prices conditional on the agent's exit, thereby driving down the profit of these agents. Therefore, the equilibrium fraction of agents that destroy value is determined by this profit and cost trade-off. 
I find that regulations that increase the entry or operating costs decrease the fraction of agents with value-destroying projects. This, however, decreases the average firm value as described above, since agents with good projects exert less effort. Therefore, regulations that are aimed at mitigating short-termism by driving out value-destroying agents might actually achieve the opposite of their goal and result in a reduction in firms' value instead. It is important to note that these regulations would improve firm value if the effort exerted by the agents with good projects did not change in response. The reason I find the opposite result is that the positive impact of fewer value-destroying agents is dominated by the moral hazard problem that becomes more severe.

This paper contributes to multiple strands of the literature. The first strand is the large literature on short-termism that primarily goes back to Narayanan (1985) and Stein (1988, 1989). In this literature, the closest papers to mine are the ones that argue short-termism might have positive effects due to some frictions (e.g., Bolton, Scheinkman, and Xiong (2006), Laux (2012), Hackbarth, Rivera, and Yong (2018), Donaldson, Malenko, and Piacentino (2020), Thakor (2021)). In contrast to these papers, the focus in my paper is how incorporating valuedestroying agents can be optimal firm value, because it strenghtens the incentives of other agents to exert more effort and create more value as a result.

Since the agent in my model can represent a blockholder, the second strand my paper is closely related to is the blockholder literature, which is broadly divided into two branches: "voice" and "exit". ${ }^{1}$ My paper falls under the voice category, where the blockholder intervenes in a firm to influence the actions or projects undertaken (e.g., Shleifer and Vishny (1986), Admati et al. (1994), Burkart et al. (1997), Cohn and Rajan (2013), Corum and Levit (2019), Corum (2020)). Under the voice category, a further subset of papers study the relation between liquidity and the blockholder's incentives to engage in an intervention (e.g., Kyle and Vila (1991), Bolton and von Thadden (1998), Kahn and Winton (1998), Maug (1998), Noe

\footnotetext{
${ }^{1}$ For surveys on voice and exit, see, e.g., Edmans (2014) and Edmans and Holderness (2017).
} 
(2002), Aghion, Bolton, and Tirole (2004), Faure-Grimaud and Gromb (2004), Back et al. (2018)). An important difference with respect to these papers is that I study the impact of incorporating a value-destroying agent on the incentives of the agent that creates value, and I show that the overall effect on the firm value can be positive.

Note that even though the last set of papers mentioned above involve liquidity, the exit literature generally refers to a different branch of the blockholder literature (and hence also incorporates a more specific concept of "exit" compared to my paper). Broadly speaking, the exit literature studies how a blockholder can influence the decisions of the manager of the firm, not through voice, but because the exit of the blockholder can reveal negative information about the firm and hence punish the manager (e.g., Admati and Pfleiderer (2009), Edmans (2009), Cvijanovic, Dasgupta, and Zachariadis (2019)). ${ }^{2}$ In contrast to these papers, in my paper, there is no other player that affects the firm value other than the agent I model, which represents the blockholder himself in the context of this literature.

\section{Setup}

In the model, there is a firm and an agent. I normalize the initial value of the firm to zero. The agent's action affects the value of the firm (I use "action" and "project" interchangeably). The agent can represent the manager or the board of the firm, as well as an entrepreneur, venture capital, private equity, or activist shareholder. The agent has $\alpha$ stake in the firm, and the agent can have either of the two types of projects: good and bad. Whether the project is good or bad is private information of the agent. The agent with the bad project always destroys firm value, and I denote the impact of the bad project on the firm value by $\Delta_{L}<0$. In contrast, the

\footnotetext{
${ }^{2}$ Some papers combine voice and exit (e.g., Edmans and Manso (2011), Dasgupta and Piacentino (2015), Song (2015), Fos and Kahn (2019), Edmans, Levit, and Reilly (2019), Levit (2019)). For other models where a blockholder's trading impacts the manager's decisions, see, e.g., Khanna and Mathews (2012) and Goldman and Strobl (2013).
} 
agent with the good project always increases the value of the firm. In particular, if he exerts effort, then the firm value increases by $\Delta_{H}>0$ but the agent incurs a cost of $c$. If he does not exert effort, then the firm value increases by $\Delta_{M}>0$. I denote the time at which the agent takes the action by $t=1$ (i.e., the "action" stage). At this stage, NPV of the project is the private information of the agent.

The main friction of the model is that it takes time for the NPV of the agent's action to be realized, and the agent can sell his stake before then. In particular, the agent can sell his stake by $t=2$ (i.e., the "exit" stage), and the NPV becomes public information at $t=3$ (i.e, the "realization" stage). At the exit stage, with probability $\phi$ the agent has to sell his stake (e.g., due to a liquidation shock or an attractive outside opportunity), and with probability $1-\phi$ the agent chooses whether to sell his stake or not. If the agent sells, the sale is observed by a market maker, who determines the stock price based on this information. For simplicity, I assume that the agent can either sell either all of his stake or none of it. ${ }^{3}$

At the beginning of the game $(t=0)$, there is also a stage where I endogenize the entry of the agent. I call this stage the "entry" stage, which consists of two components: The first component is the entry or operating cost that the agent incurs, which represents the expenses the agent needs to incur to start or run its business. Alternatively, this cost can also represent the agent's cost of taking the action (which, in this case, would be in addition to the cost $c$ that the skilled agent incurs to exert effort). In particular, the agent with bad project incurs a cost of $\kappa$ to enter, while the agent with good project incurs $\kappa-c_{s}$, where $c_{s}>0$. Here, $c_{s}$ represents the additional cost the agent with bad project incurs by entering compared to that with good project, for example, legal liability due to the value that the bad project destroys. The second component of the entry stage is the price paid by the agent to obtain his stake. In particular, I assume that the agent pays $\eta V$ per share, where $\eta \leq 1$, and $V$ represents the

\footnotetext{
${ }^{3}$ In unreported analysis, I show that the results continue to hold qualitatively even if the agent can sell any fraction of his stake and the market maker can infer the agent's sale only if the agent sells a sufficiently large fraction of his stake.
} 
market's expectation about the NPV the agent will create (again, recall that I normalize the initial value of the company to zero). Note that the reason why the market does not have a better assessment of the firm value is that the NPV of the project is private information of the agent at this stage. I assume that the supply of agents with good projects is limited and the supply of agents with bad projects are unlimited.

\section{Analysis}

I solve for the Perfect Bayesian Equilibria of the game. All proofs are in the appendix. I denote the probability that the agent with the good project exerts effort by $\rho$, the stock price upon the agent's sale decision by $P$ (which I also refer to as the "exit price"), and the fraction of agents with bad project by $q$. I start the analysis with the following preliminary lemma.

Lemma 1 For any $q \in[0,1)$, the agent with good project never exerts effort if $c>\bar{c}$ and exerts effort with positive probability if $c<\bar{c}$, where

$$
\bar{c} \equiv(1-\phi) \alpha\left(\Delta_{H}-\Delta_{M}\right)
$$

Let us understand the intuition behind Lemma 1. The payoff of the agent with good project from not exerting effort is given by

$$
\alpha\left[\phi P+(1-\phi) \max \left\{P, \Delta_{M}\right\}\right]
$$

since the payoff of the stock is $\Delta_{H}$ if the agent waits until the NPV is realized, and it is $P$ if he does not wait and chooses to exit instead. In contrast, the payoff of the agent from exerting effort is given by

$$
\alpha\left[\phi P+(1-\phi) \Delta_{H}\right]-c,
$$


since the exit price $P$ always satisfies $P \leq \Delta_{H}$ (in other words, the agent always prefers to keep his stake if he exerts effort, since the market does not fully reflect his effort at the exit stage). Therefore, the agent exerts effort only if

$$
\begin{aligned}
\alpha\left[\phi P+(1-\phi) \Delta_{H}\right]-c & \geq \alpha\left[\phi P+(1-\phi) \max \left\{P, \Delta_{M}\right\}\right] \\
& \Leftrightarrow c \leq(1-\phi) \alpha\left(\Delta_{H}-\max \left\{P, \Delta_{M}\right\}\right) .
\end{aligned}
$$

However, if $c>\bar{c}$, then (2) is never satisfied, and therefore the agent never exerts effort. This is because then, even if the exit price $P$ is so low that the agent prefers to keep his stake, he still does not exert effort because for him, it is not worth to incur the $\operatorname{cost} c$ to increase the stock payoff from $\Delta_{M}$ to $\Delta_{H}$.

For this reason, throughout the analysis in the paper, I will focus on the case where $c<\bar{c}$. Note that this restriction also ensures the first-best is that the agent with a good project

always exerts effort. In the following analysis, in Section $3.1 \mathrm{I}$ first solve the model for any given fraction $q$ of agents with bad projects, and then I endogenize $q$ in Section 3.2.

\subsection{Exogenous $q$}

I solve the model backwards. In particular, I first derive the agent's equilibrium behavior at the exit stage, and then solve for the effort level. The next lemma characterizes the agent's exit strategy in equilibrium.

Lemma 2 For any $q \in[0,1)$, in any equilibrium,

(i) The agent with bad project always exits.

(ii) If the agent with good project exerts effort, he never exits unless he has to.

(iii) If the agent with good project does not exert effort, he always exits. 
Moreover, if there are no value destroying agents (i.e., $q=0$ ), the agent with good project exerts no effort with positive probability (i.e., $\rho^{*}<1$ ).

It is relatively straightforward to understand the exit strategy of the agent if he has a good project and has exerted effort, or he has a bad project, given by parts (i) and (ii) of Lemma 2, respectively. Let us start with the former. Regardless of the fraction of agents with bad projects (that is, for any $q \in[0,1)$ ), the exit price $P$ (that is, the stock price conditional on the agent's exit) always satisfies $P^{*}>\Delta_{L}$, because there is a positive probability that the agent has a good project and is selling his stake involuntarily. However, the agent with bad project knows that the value of the firm will be $\Delta_{L}<0$ at the end due to his actions, and therefore strictly prefers to sell his stake at the exit stage.

On the other end of the spectrum, the agent with good project never exits if he has exerted effort, because the NPV is not realized at the NPV stage yet. In other words, if he is going to exit before he consequences of his effort is realized, then he has no reason to exert effort in the first place.

Note that another way to see why the agent with good project always keeps his stake if he can is to realize that the exit price $P$ always satisfies $P^{*}<\Delta_{H}$ in equilibrium. This is because the only other possibility would be $P^{*}=\Delta_{H}$, but for this to hold, it must be that all agents have good projects and they always exert effort. However, the eventual stock payoff is also $\Delta_{H}$ if the agent exerts effort, and therefore the agent gains nothing by exerting effort and keeping his stake until the realization of the NPV. For this reason, the agent would strictly prefer not exerting effort and exiting instead at the price of $P^{*}=\Delta_{H}$. Therefore, such an exit price can never incentivize the agent to exert effort, and hence the exit price $P^{*}$ has to be lower, resulting in $P^{*}<\Delta_{H}$. Importantly, this observation also reveals the inefficiency that is at the core of the paper: When there are no value destroying agents, not all agents with good projects exert effort, even though that is the first-best (due to the upperbound (1) on the cost of effort). As 
we will see in Proposition 1, the existence of value destroying agents alleviates this problem. However, before that, we also need to understand the exit strategy of the good agent that has not exerted effort, which is another importance piece of the picture.

In particular, as part (iii) in Lemma 2 describes, the agent with good project always exits if he has not exerted effort. Intuitively, this agent does not exit only if the exit price is weakly lower than the value of the firm (that is, $P \leq \Delta_{M}$ ). However, whenever this is the case, the agent knows in advance that he will keep his stake if he can, that is, he will keep it with probability $1-\phi$. Since he has a stake of $\alpha$ in the firm, the additional payoff he gets from exerting effort is $(1-\phi) \alpha\left(\Delta_{H}-\Delta_{M}\right)$ at a cost of $c . c<\bar{c}$ and (1) imply that the agent is strictly better off by exerting effort to increase the firm value from $\Delta_{M}$ to $\Delta_{H}$. Therefore, if the exit price is indeed low such that $P \leq \Delta_{M}$, the agent with good project will always exert effort. In other words, this agent does not exert effort only if the exit price is high $\left(P>\Delta_{M}\right)$, and then it is always profitable for him to exit.

Note that an implication of Lemma 2 is that as a function of $\rho^{*}$ (that is, the probability with which the agent with good project exerts effort), the exit price $P^{*}$ in any equilibrium is given by

$$
P^{*}\left(\rho^{*}\right)=\frac{(1-q) \rho^{*} \phi \Delta_{H}+(1-q)\left(1-\rho^{*}\right) \Delta_{M}+q \Delta_{L}}{1-(1-q) \rho^{*}(1-\phi)},
$$

In turn, the exit price $P$ determines the agent's incentives to exert effort. In particular, the agent is indifferent between exerting effort and keeping staking vs. not exerting effort and exiting if and only if

$$
(1-\phi) P=(1-\phi) \Delta_{H}-\frac{c}{\alpha}
$$

because the agent has to exit with probability $\phi$, and he has a choice between exiting and not with only probability $1-\phi$. Plugging (4) in (3) yields the fixed point (6) given in Proposition 1 below, which is the equilibrium level of effort $\rho^{*}$ if it does not exceed 1 . Indeed, if $\rho^{*}$ given by (6) does not exceed 1 , then the resulting $P^{*}$ satisfies the equality (4). If $\rho^{*}$ were any larger, then 
(3) implies that the resulting $P^{*}$ would be strictly larger. ${ }^{4}$ Intuitively, the market incorporates the higher effort of the agent in the exit price, since the agent that exerts effort still has to exit with probability $\phi$. As a result, the agent would strictly prefer not exerting effort and exiting instead, since the exit price would be too tempting for the agent. In contrast, if $\rho^{*}$ were any smaller, then $P^{*}$ would be too low, and hence the agent would strictly prefer to keep his stake if he can. Then, since the agent would rationally anticipate that he will keep his stake, he would exert effort.

The situation where the agent with good project does not always exert effort (that is, $\rho^{*}<1$ ) arises if and only if $q$ (i.e., the fraction of agents who destroy value) is not too large, that is $q<\underline{q}$. The formal expression for the threshold $\underline{q}$ is given by (5) in Proposition 1 below. Intuitively, as mentioned above, if the agent with good project always exerted effort then the exit price $P$ would become too large to incentivize the agent to exert effort. Importantly, in this region, as $q$ increases $\rho^{*}$ increases as well. The reason is that if there are more value destroying agents (i.e., higher $q$ ), they have a more negative impact on the exit price $P$. In turn, lower exit price incentivizes the agent with good project to keep his stake until the realization of the NPV. Therefore, the agent internalizes the impact of his actions on the firm more, and exerts more effort. In particular, to counteract the negative impact of the value destroying agents, the agent with good project has to exert more effort for the exit price to reach its original level, given by (4).

In contrast, if $q$ is larger, then for any effort level the resulting $P^{*}$ is always low (in particular, in the lower then the level (4) implies), and therefore the agent with good project is always incentivized to exert effort (i.e., $\rho^{*}=1$ ). This threshold of $q$ is formally given in Proposition 1 below, which summarizes what is described above and characterizes the equilibrium effort

\footnotetext{
${ }^{4}$ Indeed, $P^{*}$ given by (3) is strictly increasing in $\rho^{*}$ if and only if $\Delta_{M}<\phi \Delta_{H}+P^{*}(1-\phi)$. This condition always holds if $\rho^{*}$ given by (6) does not exceed 1 , since the latter implies $P^{*}>\Delta_{M}$. This is because if it were $P^{*} \leq \Delta_{M}$, then the agent with good project would always exert effort (that is, it would be $\rho^{*}=1$ ) due to arguments made above when explaining part (iii) of Lemma 2.
} 
level $\rho^{*}$ and exit price $P^{*}$.

Proposition 1 Let

$$
\underline{q} \equiv \frac{\frac{\phi}{1-\phi} \frac{c}{\alpha}}{\Delta_{H}-\Delta_{L}-\frac{c}{\alpha}},
$$

where $q \in(0,1)$. For any $q$, the equilibrium is unique, and in equilibrium:

(i) If $q<\underline{q}$, then

$$
\begin{aligned}
\rho^{*} & =\frac{1}{1-q}\left(1+\frac{q\left(\Delta_{M}-\Delta_{L}\right)-\frac{\phi}{1-\phi} \frac{c}{\alpha}}{\Delta_{H}-\frac{c}{\alpha}-\Delta_{M}}\right) \in(0,1), \\
P^{*} & =\Delta_{H}-\frac{1}{1-\phi} \frac{c}{\alpha}
\end{aligned}
$$

Moreover, $\rho^{*}$ is strictly increasing in $q$.

(ii) If $q \geq \underline{q}$, then $\rho^{*}=1$, and

$$
P^{*}=\frac{(1-q) \phi \Delta_{H}+q \Delta_{L}}{1-(1-q)(1-\phi)}
$$

As mentioned earlier, if $q$ is not too large, then $\rho^{*}$ increases as $q$ increases. In other words, as the number of value destroying agents increases, the agents with good projects exert more effort. However, which of these effects dominate for the value of the firm? Denoting the unconditional firm value by $V$, and noting that it is given by

$$
V(\rho ; q)=(1-q) \rho \Delta_{H}+(1-q)(1-\rho) \Delta_{M}+q \Delta_{L},
$$

the next proposition formalizes that the higher effort dominates the negative impact of value destruction and the firm value is maximized at $q=q$.

Proposition $2 V^{*}$ is strictly increasing in $q$ if $q<\underline{q}$, attains its unique maximum at $q=\underline{q}$, and it is strictly decreasing in $q$ if $q \geq \underline{q}$. Moreover, $V^{*}(\underline{q})$ strictly increases as $\Delta_{L}$ decreases. 
As $q$ increases, why does the positive effect of more effort by agents that have good projects dominates the negative effect of more value destruction by the other agents? As the fraction of value-destroying agents increases, the agents with positive NPV projects increase their effort to a certain point. In particular, their higher effort increases the average stock price upon their exit to the point given by (4), where they become once again indifferent between exerting effort (and keeping their stake) vs. not exerting effort (and selling their stake). The critical observation is that the impact of higher effort of the agent has more positive impact on the unconditional average value of the firm $V$ compared to the average stock price $P$ conditional on the exit of the agent. This is because upon the exit of the agent, the market assigns a lower probability that the agent has exerted effort, since such an agent does not exit unless he has to. Therefore, as the agent with positive NPV exerts more effort and the agent's exit price $P$ reaches its original level, this higher effort results in even a larger increase in the unconditional firm value $V$ and pushes it up beyond its old level. This is how the average firm value increases as the fraction of value-destroying agents increases, and it holds all the way until this fraction is sufficiently high (i.e., $q=q$ ) so that the agents with positive NPV projects always exert effort and keep their stake.

However, beyond $\underline{q}$, increasing the ratio of value destroying agents does not provide any additional benefit but only harm to the firm value. Intuitively, the agents with good projects always exert effort at this point, and therefore including more value destroying agents does not make them exert more effort. As a result, the average firm value $V^{*}$ decreases as $q$ increases further, and hence the maximum firm value is attained at $q=\underline{q}$, which I denote by $V^{*}(\underline{q})$.

Another interesting result in Proposition 2 is that the maximum firm value that can be achieved across all $q$ (i.e., $\left.V^{*}(\underline{q})\right)$ actually increases as $\Delta_{L}$ decreases. While this result may seem counter-intuitive, the reason is as follows. At $q=\underline{q}$, the agent with good project always exerts effort, and hence exits with only probability $\phi$. However, the value destroying agent always exits. Therefore, the unconditional probability that the agent exits is given by $\underline{q}+\phi(1-\underline{q})$, 
and the average value of the firm conditional on the agent's exit is equal to exit price, $P^{*}$. On the other hand, the agent does not exit with an unconditional probability $(1-\underline{q})(1-\phi)$, that is, the agent has a good project and does not have to exit. Conditional on this event, the firm value is given by $\Delta_{H}$. Combining, the unconditional firm value $V^{*}(\underline{q})$ can be summarized as

$$
V^{*}(\underline{q})=[\underline{q}+\phi(1-\underline{q})] P^{*}+(1-\underline{q})(1-\phi) \Delta_{H},
$$

where $P^{*}$ is given by $(7) .{ }^{5}$ Importantly, (7) does not depend on $\Delta_{L}$ or $\underline{q}$, and therefore the expression given by (10) is strictly increases as $\underline{q}$ decreases.

In other words, the unconditional firm value $V^{*}(\underline{q})$ is given by a weighted average of the firm value conditional on the agent's exit and conditional on agent keeping his stake. Importantly, the value of the firm conditional on either of these events do not change with $\underline{q}$ or $\Delta_{L}$. This is because if the agent is not exiting, then it must be the agent that has a good project, and therefore the firm value must be $\Delta_{H}$. Similarly, if the agent is exiting, then the conditional expected firm value is given by the price that keeps the agent with good project indifferent between exerting effort and not, that is, (4). ${ }^{6}$ In other words, the conditional average firm value in this case does not have anything to do with the value destroying agent per se.

However, the probabilities of these two events (the agent exiting vs. not exiting) does depend on $\underline{q}$. Importantly, the probability of no exit increases as $\underline{q}$ decreases, since the value destroying agent exits more frequently than the agent that has exerted effort. Therefore, as $\underline{q}$ decreases, the unconditionitional firm value (10) increases. Importantly, the reason why $V^{*}(\underline{q})$ increases as $\Delta_{L}$ decreases is that $\underline{q}$ decreases as a result. Intuititively, $\underline{q}$ is the smallest value of $q$ for which the agent with good project always exerts effort. The agent is motivated to exert effort only if the exit price is low, and value-destroying agent has a more negative impact on the exit price if he destroys even more value. Therefore, if $\Delta_{L}$ is smaller, full effort is reached

\footnotetext{
${ }^{5}$ Plugging (5) in (8) reveals that (7) applies at $q=q$ as well.

${ }^{6}$ In particular, $q$ is the highest value of $q$ such that this indifference holds.
} 
at a smaller $q$, implying that $\underline{q}$ is smaller. To put it differently, if the agent with a bad project destroys more value, less of them are needed to motivate all of the agents with good projects to separate from them and keep their stake, in turn inducing them to exert effort.

Remark. No result presented so far in the analysis (Section 3) depends on the assumption $\Delta_{L}<0$, including the proofs of all the lemmas and propositions so far. Indeed, the only needed assumption for all these results is $\Delta_{L}<\Delta_{M}$. Note that even if $\Delta_{L} \in\left[0, \Delta_{M}\right)$, the agent with the bad project still destroys firm value, because it is replacing the agent with good project who always creates a firm value of $\Delta_{M}$, which is strictly larger than $\Delta_{L}$. In fact, throughout the analysis, only Proposition 4 below utilizes the assumption $\Delta_{L}<0$ to simplify the exposition, and in an unreported analysis I show that it continues to hold qualitatively without that assumption as well.

\subsubsection{Career concerns and agent's "skill"}

While the arguments above abstract from career concerns, at this point it is worthwhile to mention that the results would not get weaker (and instead sometimes get stronger) if career concerns were incorporated in my model as well. First of all, it is important to note the modeling of the agent in this paper captures more than the skillfulness of the agent. In particular, I do not make any assumption about the time-series correlation of the quality of an agent's project. If the quality of the agents' projects now and in the future are independent, then incorporating career concerns would have no impact on the agents' incentives, since the market wouldn't make any inference from the NPV of the agent's current project for the NPV of his future projects (for simplicity, my model consists of a single stage where an agent implements a project only once, but it could be easily extended to a repeated game setting where the agent can implement different projects over time, and the results would continue to hold). In contrast, if there is positive correlation between the agent's projects over time (that is, the agent has a level of "skill"), then adding career concerns on top of my model would 
make my results stronger. This is because as the fraction of agents with negative NPV projects increase, the incentives of the "skilled" agent to keep his stake would increase so that he can separate from the "unskilled" agent, in turn strengthening his incentives to exert effort.

However, this does not imply that the main result (i.e., that a higher ratio of valuedestroying agents can increase average firm value) would necessarily continue to hold in an alternative model where career concerns replace short-termism (which stems from the opportunity for the agent to sell the stake before the NPV is realized). This is because, arguably, it is more difficult to quantify the impact of the hightened career concerns on the incentives of the agent to exert effort, and therefore it is not clear whether this positive effect would dominate the negative impact of more value-destroying agents. Moreover, as described above, career concerns have no effect on the agent's incentives to exert effort to begin with in cases where there is no inference to made about the agent's skill. In contrast, my model relies on the effect of the stock price, which is likely a more objective measure in many cases.

That said, the results of my model continue to hold even if the agent has a level of "skill" (i.e., there is a positive correlation between the quality of an agent's projects over time). Therefore, an implication of the model is that an agent that has proven his skill over time might start creating less value compared to an agent that hasn't proved his skill yet, even if there are no career concerns involved.

\subsubsection{When a principal chooses between agents}

Regardless of whether there is any element of skill or career concerns, the model also has interesting implications for the case when there is a principal in charge of choosing the agent. First, if the principal is facing two different agents' proposals at the same time (for example, shareholders of a firm voting for the board of directors vs. an activist in a proxy fight), it might be optimal for the principal to follow the proposal by the agent who is more likely to destroy value. 
Second, whenever there is a principal that selects the agent (e.g., the board choosing a $\mathrm{CEO}$ ), it might be optimal for the principal to be not too expert in identifying whether the agent has positive vs. negative NPV projects (or alternatively, whether the agent is skilled vs. unskilled, if there is a skill component). This is because if the principal can perfectly distinguish between these agents, then she strictly prefers the agent that creates value. However, as described above, such an agent might create less value compared to an agent that destroys value with some probability (where this probability is denoted by $q$ in the model). Interestingly, this is true even if the market itself cannot make such a distinction: At the end of the day, the market knows that the principal will act in her best interest, and choose the agent accordingly.

In the rest of the analysis, for simplicity I continue abstracting from such a principal who can identify the quality of an agent's project in advance.

\section{$3.2 \quad$ Endogenous $q$}

In this section, I endogenize the fraction of agents that implement value-destroying projects, since doing so enables studying effects of regulations that might impact this fraction and the average quality of projects as a result. In particular, I assume that agents have some entry or operating costs, which represent the expenses the agent needs to incur to start or run his business. Alternatively, this cost can also represent the agent's cost of implementing his project (which, in this case, would be in addition to the cost that the skilled agent incurs to exert effort). In particular, the agent with bad project incurs a cost of $\kappa$ to enter, while the agent with good project incurs $\kappa-c_{s}$, where $c_{s}>0$. Here, $c_{s}$ represents the additional cost the agent with bad project incurs by entering compared to that with good project, for example, legal liability due to the value that the bad project destroys.

In addition, I also add the flexibility to the model to account for the price paid by the agent to obtain his stake. In particular, I assume that the agent pays $\eta V$ per share, where $\eta \leq 1$, and 
$V$ represents the market's expectation about the NPV the agent will create (again, recall that I normalize the initial value of the company to zero). Note that the reason why the market does not have a better assessment of the firm value is that the NPV of the project is private information of the agent at this stage. I assume that the supply of agents with good projects is limited and the supply of agents with bad projects are unlimited.

I start the analysis by showing a preliminary result. In particular, as described in the following lemma, there exits a threshold $\hat{\kappa}$ such that if the entry cost is higher than this threshold then no agent enters, and if it is lower then the agents with good project always enters. This is because the profit of the value-destroying agent from entering is lower than that of the agent with good project. Note that $P^{*}(q)$ and $V^{*}(q)$ in the following lemma are functions of $q$, which represent the exit price and unconditional expected value of the firm, respectively.

Lemma 3 Let

$$
\hat{\kappa} \equiv c_{s}+P^{*}(0)-\eta V^{*}(0)
$$

Then,

(i) No agent enters if $\kappa>\hat{\kappa}$.

(ii) All of the agents with good projects enter if $\kappa<\hat{\kappa}$.

Due to Lemma 3, I assume that $\kappa<\hat{\kappa}$ in the remainder of the analyis.

A value-destroying agent can make profit because he can sell his stake before the NPV is realized. In particular, he strictly prefers to enter if $\kappa<P^{*}-\eta V^{*}$ (and is indifferent if $\kappa=P^{*}-$ $\eta V^{*}$ and strictly prefers not to enter otherwise). However, as more agents implement negative NPV projects, the market incorporates this expectation into the stock prices conditional on the agent's exit, thereby driving down the profit of these agents (in other words, $P^{*}(q)-\eta V^{*}(q)$ 
decreases). Therefore, the fraction of agents that destroy value is determined such that the net profit of the agent in equilibrium is zero. The next proposition formalizes this result.

Proposition 3 Suppose that $\kappa>(1-\eta) \Delta_{L}$. Then, the equilibrium is unique, and it is given by

$$
q^{*}= \begin{cases}0, & \text { if } \kappa \geq P^{*}(0)-\eta V^{*}(0) \\ q \in(0,1] \text { s.t. } \kappa=P^{*}(q)-\eta V^{*}(q), & \text { otherwise. }\end{cases}
$$

Moreover, $P^{*}(q)-\eta V^{*}(q)$ strictly decreases w.r.t $q$ if $P^{*}(q)-\eta V^{*}(q)>(1-\eta) \Delta_{L}$.

Now that I have characterized the equilibrium, we can turn our attention to study the effects of the regulations that increase the entry or operating costs by doing a comparative static w.r.t. $\kappa$. The next proposition characterizes this comparative static.

(Mention the following before the next proposition: The unskilled agent strictly prefers to enter if $\kappa<P^{*}-\eta V^{*}$, is indifferent if $\kappa=P^{*}-\eta V^{*}$, and strictly prefers not to enter otherwise.)

Proposition 4 Suppose that $\Delta_{L}<0$ and $\kappa \geq 0$. Then, the equilibrium is unique, and in particular,

(i) If $\eta \geq P^{*}(0) / V^{*}(0)$, then $q^{*}=0$ in any equilibrium.

(ii) If $\eta<P^{*}(0) / V^{*}(0)$, then letting

$$
\begin{aligned}
\bar{\kappa} & \equiv P^{*}(0)-\eta V^{*}(0), \\
\underline{\kappa} & \equiv \max \left\{0, P^{*}(\underline{q})-\eta V^{*}(\underline{q})\right\},
\end{aligned}
$$

$0 \leq \underline{\kappa}<\bar{\kappa}$ holds, and as $\kappa$ decreases,

(a) If $\kappa>\bar{\kappa}$, then $q^{*}=0$ and $V^{*}$ does not change. 
(b) If $\kappa \in(\underline{\kappa}, \bar{\kappa}]$, then $q^{*}$ and $V^{*}$ strictly increase. Moreover, $V^{*}$ attains its global maximum w.r.t. $\kappa \in[0, \infty)$ at $\kappa=\underline{\kappa}$.

(c) If $\kappa \in[0, \underline{\kappa}], q^{*}$ strictly increases and $V^{*}$ strictly decreases.

If the premium an agent pays when entering reflects a high percentage $\eta$ of the value of the firm $V$, then no value-destroying agent enters since it is too costly to do. However, if $\eta$ is sufficiently low, then a positive number of value-destroying agents enter, and I find that reducing $\kappa$ increases the fraction of agents with value-destroying projects. This, however, increases the average firm value as described in Section 3.1, since agents with good projects exert more effort. Therefore, regulations that are aimed at mitigating short-termism by driving out value-destroying agents might actually achieve the opposite of their goal by making the moral hazard problem much worse, thereby resulting in a reduction in firms' value instead. It is important to note that these regulations would improve firm value if the effort exerted by the agents with good projects did not change in response. The reason I find the opposite result is that the positive impact of fewer value-destroying agents is dominated by the moral hazard problem that becomes more severe.

\section{Conclusion}

In this paper, I study a model of short-termism where a firm's value is affected by the actions of an agent, who can represent the manager or the board, as well as an entrepreneur, venture capital, private equity, or activist shareholder. The agent either has a project with positive NPV and can further increase the NPV by exerting effort, or has a project that destroys value. The agent has a stake in the company and can liquidate it before the NPV of his actions are realized by the market. This ability to exit creates short-term incentives for the agent to not exert effort, as well as opportunities to profit even if he is destroying value. I find that replacing 
a fraction of agents that have positive NPV projects with value-destroying agents can increase average firm value, because it motivates the former agent to work harder, and this effect can dominate the added value destruction. Moreover, this result also holds under endogenous entry of agents: Reducing the entry or operating costs for the agents can increase average firm value and gross value destruction simultaneously, even though the fraction of agents with positive NPV projects decreases. Therefore, regulations that aim mitigating short-termism by curbing value destruction can actually yield opposite results and reduce average firm value instead. 


\section{References}

[1] Admati, Anat R., and Paul Pfleiderer, 2009, The "Wall Street Walk" and shareholder activism: exit as a form of voice, The Review of Financial Studies 22, 2245-2285.

[2] Admati, Anat R., Paul Pfleiderer, and Josef Zechner, 1994, Large shareholder activism and financial market equilibrium, Journal of Political Economy 102, 1097-1130.

[3] Aghion, Philippe, Patrick Bolton, and Jean Tirole, 2004, Exit Options in Corporate Finance: Liquidity versus Incentives, Review of Finance 8, 327-53.

[4] Back, Kerry, Pierre Collin-Dufresne, Tao Li, and Alexander Ljungqvist, 2018, Activism, strategic trading, and liquidity, Econometrica 86, 1431-1463.

[5] Bolton, Patrick, Jose Scheinkman, and Wei Xiong, 2006, Executive compensation and short-termist behaviour in speculative markets, The Review of Economic Studies 73, 577610 .

[6] Bolton, Patrick, and E. Ludwig von Thadden, 1998, Blocks, liquidity, and corporate control, Journal of Finance 53, 1-25.

[7] Burkart, M., D. Gromb, and F. Panunzi, 1997, Large Shareholders, Monitoring and the Value of the Firm, Quarterly Journal of Economics 112, 693-728.

[8] Cohn, Jonathan B., and Uday Rajan, 2013, Optimal corporate governance in the presence of an activist investor, Review of Financial Studies 26, 985-1020.

[9] Corum, Adrian Aycan, Activist Settlements, 2020, Working paper.

[10] Corum, Adrian Aycan, and Doron Levit, 2019, Corporate control activism, Journal of Financial Economics 133, 1-17.

[11] Cvijanovic, Dragana, Amil Dasgupta, and Konstantinos E. Zachariadis, 2019, The Wall Street Stampede: Exit As Governance with Interacting Blockholders, Working paper.

[12] Dasgupta, Amil and Giorgia Piacentino, 2015, The Wall Street Walk When Blockholders Compete For Flows, Journal of Finance 70 (6), 2853-2896.

[13] Donaldson, Jason, Nadya Malenko, and Giorgia Piacentino, 2020, Deadlock on the board, The Review of Financial Studies 33, 4445-4488.

[14] Edmans, Alex, 2009, Blockholder trading, market efficiency, and managerial myopia, Journal of Finance 64, 2481-513.

[15] Edmans, Alex, 2014, Blockholders and corporate governance, Annual Review of Financial Economics 6, 23-50. 
[16] Edmans, Alex, Doron Levit, and Devin Reilly, 2019, Governance under common ownership, Review of Financial Studies 32, 2673-2719.

[17] Edmans, Alex, and Clifford G. Holderness, 2017, Blockholders: A survey of theory and evidence in Handbook of corporate governance, ed. Benjamin Hermalin and Michael Weisbach (New York: Elsevier/North-Holland).

[18] Edmans, Alex, and Gustavo Manso, 2011, Governance Through Trading and Intervention: A Theory of Multiple Blockholders, Review of Financial Studies 24, 2395-2428.

[19] Faure-Grimaud, A., and D. Gromb, 2004, Public Trading and Private Incentives, Review of Financial Studies 17, 985-1014.

[20] Fos, Vyacheslav, and Charles Kahn, 2019, Governance through threats of intervention and exit, Working Paper.

[21] Goldman Eitan, and Gunther Strobl, 2013, Large shareholder trading and the complexity of corporate investments, Journal of Financial Intermediation 22, 106-122.

[22] Hackbarth, Dirk, Alejandro Rivera, and Tak-Yuen Wong, 2018, Optimal short-termism, Working paper.

[23] Kahn, C., and A. Winton, 1998, Ownership Structure, Speculation, and Shareholder Intervention, Journal of Finance, 53, 99-129.

[24] Khanna, Naveen, and Richmond D. Mathews, 2012, Doing Battle With Short Sellers: The Conflicted Role of Blockholders in Bear Raids, Journal of Financial Economics 106, $229-46$.

[25] Kyle, Albert S., and Jean-Luc Vila, 1991, Noise trading and takeovers, Rand Journal of Economics 22, 54-71.

[26] Laux, Volker, 2012, Stock option vesting conditions, CEO turnover, and myopic investment, Journal of Financial Economics 106, 513-526.

[27] Levit, Doron, 2019, Soft shareholder activism, Review of Financial Studies 32, 2775-2808.

[28] Maug, Ernst, 1998, Large Shareholders as Monitors: Is There a Tradeoff Between Liquidity and Control?, Journal of Finance 53, 65-98.

[29] Narayanan, M. P., 1985, Managerial incentives for short-term results, The Journal of Finance 40, 1469-1484.

[30] Noe, T., 2002, Investor Activism and Financial Market Structure, Review of Financial Studies 15, 289-318. 
[31] Shleifer, Andrei, and Robert W. Vishny, 1986, Large shareholders and corporate control, Journal of Political Economy 94, 461-488.

[32] Song, Fenghua, 2017, Blockholder short-term incentives, structures, and governance, Working Paper.

[33] Stein, Jeremy C., 1988, Takeover threats and managerial myopia. Journal of Political Econonomy 96, 61-80.

[34] Stein, Jeremy C., 1989, Efficient capital markets, inefficient firms: a model of myopic corporate behavior, Quartely Journal of Economics 104, 655-669.

[35] Thakor, Richard, 2021, Short-termism, managerial Talent, and firm Value, The Review of Corporate Finance Studies, forthcoming.

[36] Xiong, 2020, Managerial short-termism and market competition, Working paper. 


\section{A Proofs}

Proof of Lemma 1. Note that the payoff of the agent with good project from exerting effort is given by

$$
\alpha\left[\phi P+(1-\phi) \max \left\{P, \Delta_{H}\right\}\right]-c,
$$

while his payoff from not exerting effort is given by

$$
\alpha\left[\phi P+(1-\phi) \max \left\{P, \Delta_{M}\right\}\right]
$$

Therefore, the agent exerts effort only if

$$
\begin{aligned}
\alpha\left[\phi P+(1-\phi) \max \left\{P, \Delta_{H}\right\}\right]-c & \geq \alpha\left[\phi P+(1-\phi) \max \left\{P, \Delta_{M}\right\}\right] \\
& \Leftrightarrow \frac{1}{1-\phi} \frac{c}{\alpha} \leq \max \left\{P, \Delta_{H}\right\}-\max \left\{P, \Delta_{M}\right\},
\end{aligned}
$$

and the agent exerts effort if the inequality in (12) holds strictly.

Suppose that $c>\bar{c}$. Since $P \leq \Delta_{H}$ is always satisfied, the LHS of (12) is weakly smaller than $\Delta_{H}-\Delta_{M}$, and therefore (1) and $c>\bar{c}$ imply that (12) is never satisfied. As a result, the agent never exerts effort.

Suppose that $c<\bar{c}$. There are two cases to consider: If $P \leq \Delta_{M}$, then (12) implies that the agent with the good project always exerts effort. In contrast, if $P>\Delta_{M}$, then it must be that the agent with good project is exerting effort with positive probability, since otherwise $P \leq \Delta_{M}$ would have to be satisfied, yielding a contradiction.

Proof of Lemma 2. Throughout the proof, I refer to the agent with good project as the "good agent" and the agent with bad project as the "bad agent" to simplify the language.

First, note that the bad agent always exits, because $P^{*}>\Delta_{L}$ whenever $q<1$ since $\phi>0$. Even in the corner case of $q=1$, the bad agent weakly prefers to exit since $P^{*}=\Delta_{L}$.

Second, I show that the good agent that has exerted effort never exits unless he has to. 
Note that for this result, it is sufficient to show that $P^{*}<\Delta_{H}$. Suppose this is not the case. Then, it must be that $P^{*}=\Delta_{H}$ and $\rho^{*}=1$. However, then the good agent strictly prefers to exit and not to exert effort, yielding a contradiction with $P^{*}=\Delta_{H}$.

Note that the above step also shows that it must be $\rho^{*}<1$ if $q=0$, because otherwise it would yield a contradiction with $P^{*}<\Delta_{H}$. This proves the last statement of the lemma.

Third, I show that the good agent always exits if he has not exerted effort. There are two cases to consider. First, suppose that $P^{*}>\Delta_{M}$. Then, the agent strictly prefers exiting over not exiting. Second, suppose that $P^{*} \leq \Delta_{M}$. However, then the maximum payoff the good agent will get if he doesn't exert effort is given by $\alpha\left[\phi P^{*}+(1-\phi) \max \left\{P^{*}, \Delta_{M}\right\}\right]$. In contrast, if he exerts effort and does not exit, then he gets a payoff of $\alpha\left[\phi P^{*}+(1-\phi) \Delta_{H}-c\right]$. (1) implies that the latter is strictly larger, concluding the proof.

Proof of Proposition 1. Throughout the proof, I refer to the agent with good project as the "good agent" and the agent with bad project as the "bad agent" to simplify the language. Note that $\underline{q} \in(0,1)$ because $(1)$ implies that $0<\Delta_{H}-\frac{1}{1-\phi} \frac{c}{\alpha}-\Delta_{L}$.

Note that by Lemma 2, in any equilibrium if the good agent exerts effort, then he never exits and gets a payoff of $\alpha\left[\phi P^{*}+(1-\phi) \Delta_{H}-c\right]$, and in contrast if he does not exert effort, then he gets a payoff of $\alpha\left[\phi P^{*}+(1-\phi) \max \left\{P^{*}, \Delta_{M}\right\}\right]$. Therefore, since (1) implies that $(1-\phi) \Delta_{H}-c>(1-\phi) \Delta_{M}$, the best response of the good agent (that is, the probability $\rho$ that he exerts effort) as a function of the exit price $P$ is given by

$$
\rho(P)= \begin{cases}0, & \text { if } P>\Delta_{H}-\frac{1}{1-\phi} \frac{c}{\alpha} \\ \in[0,1], & \text { if } P=\Delta_{H}-\frac{1}{1-\phi} \frac{c}{\alpha} \\ 1, & \text { if } P<\Delta_{H}-\frac{1}{1-\phi} \frac{c}{\alpha}\end{cases}
$$

Suppose that $q<\underline{q}$. Then, $P^{*}>\Delta_{H}-\frac{1}{1-\phi} \frac{c}{\alpha}$ if $\rho^{*}=1$, and $P^{*} \leq \Delta_{M}<\Delta_{H}-\frac{1}{1-\phi} \frac{c}{\alpha}$ if $\rho^{*}=0$. Therefore, it must be that $\rho^{*} \in(0,1)$, and hence $P^{*}=\Delta_{H}-\frac{1}{1-\phi} \frac{c}{\alpha}$. In turn, this yields $(6)$, 
since plugging $(3)$ in $P^{*}$ yields

$$
\begin{aligned}
\Delta_{H}-\frac{1}{1-\phi} \frac{c}{\alpha} & =P^{*}=\frac{(1-q) \rho\left[\phi \Delta_{H}-\Delta_{M}\right]+(1-q) \Delta_{M}+q \Delta_{L}}{1-(1-q) \rho(1-\phi)} \\
& \Leftrightarrow\left(\Delta_{H}-\frac{1}{1-\phi} \frac{c}{\alpha}\right)[1-(1-q) \rho(1-\phi)]=(1-q) \rho\left[\phi \Delta_{H}-\Delta_{M}\right]+(1-q) \Delta_{M}+q \Delta_{L} \\
& \Leftrightarrow \rho^{*}=\frac{1}{1-q} \frac{\left(\Delta_{H}-\frac{1}{1-\phi} \frac{c}{\alpha}\right)-(1-q) \Delta_{M}-q \Delta_{L}}{(1-\phi)\left(\Delta_{H}-\frac{1}{1-\phi} \frac{c}{\alpha}\right)+\left[\phi \Delta_{H}-\Delta_{M}\right]} \\
& \Leftrightarrow \rho^{*}=\frac{1}{1-q} \frac{\Delta_{H}-\frac{1}{1-\phi} \frac{c}{\alpha}-(1-q) \Delta_{M}-q \Delta_{L}}{\Delta_{H}-\frac{c}{\alpha}-\Delta_{M}} \\
& \Leftrightarrow \rho^{*}=\frac{1}{1-q} \frac{\Delta_{H}-\frac{c}{\alpha}-\frac{\phi}{1-\phi} \frac{c}{\alpha}-\Delta_{M}+q \Delta_{M}-q \Delta_{L}}{\Delta_{H}-\frac{c}{\alpha}-\Delta_{M}} \\
& \Leftrightarrow \rho^{*}=\frac{1}{1-q}\left(1+\frac{q\left(\Delta_{M}-\Delta_{L}\right)-\frac{\phi}{1-\phi} \frac{c}{\alpha}}{\Delta_{H}-\frac{c}{\alpha}-\Delta_{M}}\right)
\end{aligned}
$$

Note that $\rho^{*}>0$ since

$$
0<1+\frac{q\left(\Delta_{M}-\Delta_{L}\right)-\frac{\phi}{1-\phi} \frac{c}{\alpha}}{\Delta_{H}-\frac{c}{\alpha}-\Delta_{M}} \Leftrightarrow 0<\frac{\Delta_{H}-\frac{1}{1-\phi} \frac{c}{\alpha}-\Delta_{M}+q\left(\Delta_{M}-\Delta_{L}\right)}{\Delta_{H}-\frac{c}{\alpha}-\Delta_{M}},
$$

which holds since $\Delta_{H}-\frac{1}{1-\phi} \frac{c}{\alpha}>\Delta_{M}$ by (1) and $\Delta_{M}>\Delta_{L}$. Moreover, also note that $\rho^{*}<1$ if $q<\underline{q}$, because

$$
\rho^{*}<1 \Leftrightarrow \frac{1}{1-q}\left(1+\frac{q\left(\Delta_{M}-\Delta_{L}\right)-\frac{\phi}{1-\phi} \frac{c}{\alpha}}{\Delta_{H}-\frac{c}{\alpha}-\Delta_{M}}\right)<1 \Leftrightarrow q<\frac{\frac{\phi}{1-\phi} \frac{c}{\alpha}}{\Delta_{H}-\frac{c}{\alpha}-\Delta_{L}}=\underline{q}
$$

Moreover, also note that $\underline{q} \in(0,1)$ because (1) implies that

$$
0<\Delta_{H}-\frac{1}{1-\phi} \frac{c}{\alpha}-\Delta_{M}<\Delta_{H}-\frac{1}{1-\phi} \frac{c}{\alpha}-\Delta_{L}
$$

Suppose that $q \geq \underline{q}$. Then, to show that in equilibrium it must be $\rho^{*}=1$, (13) implies that it is sufficient to show that $P^{*}<\Delta_{H}-\frac{1}{1-\phi} \frac{c}{\alpha}$ if $\rho^{*}<1$, and $P^{*} \leq \Delta_{H}-\frac{1}{1-\phi} \frac{c}{\alpha}$ if $\rho^{*}=1$. In turn, 
since $\Delta_{H}-\frac{1}{1-\phi} \frac{c}{\alpha}>\Delta_{M}$ by (1), it is sufficient to show that $P^{*} \leq \Delta_{H}-\frac{1}{1-\phi} \frac{c}{\alpha}$ if $\rho^{*}=1$, and $P$ (which is given by (3)) is strictly increasing in $\rho$ if $P \geq \Delta_{M}$. To see that $P^{*} \leq \Delta_{H}-\frac{1}{1-\phi} \frac{c}{\alpha}$ if $\rho^{*}=1$, note that

$$
\begin{aligned}
P^{*} & =\frac{(1-q) \phi \Delta_{H}+q \Delta_{L}}{1-(1-q)(1-\phi)} \leq \Delta_{H}-\frac{1}{1-\phi} \frac{c}{\alpha} \\
& \Leftrightarrow(1-q)(1-\phi)\left(\Delta_{H}-\frac{1}{1-\phi} \frac{c}{\alpha}\right)+(1-q) \phi \Delta_{H}+q \Delta_{L} \leq\left(\Delta_{H}-\frac{1}{1-\phi} \frac{c}{\alpha}\right) \\
& \Leftrightarrow q>\frac{\frac{\phi}{1-\phi} \frac{c}{\alpha}}{\Delta_{H}-\frac{c}{\alpha}-\Delta_{L}}=\underline{q} .
\end{aligned}
$$

And finally, to see that $P$ is strictly increasing in $\rho$ if $P \geq \Delta_{M}$, note that $P \geq \Delta_{M}$ implies that

$$
\begin{aligned}
\Delta_{M} & \leq P \Leftrightarrow \Delta_{M} \leq \frac{(1-q) \rho \phi \Delta_{H}+(1-q)(1-\rho) \Delta_{M}+q \Delta_{L}}{1-(1-q) \rho(1-\phi)} \\
& \Leftrightarrow 0 \leq(1-q) \rho \phi \Delta_{H}+q \Delta_{L}+(1-q) \rho(1-\phi) \Delta_{M}+(1-q)(1-\rho) \Delta_{M}-\Delta_{M} \\
& \Leftrightarrow 0 \leq(1-q) \rho \phi \Delta_{H}+q \Delta_{L}-(1-q) \rho \phi \Delta_{M}+(1-q) \Delta_{M}-\Delta_{M}
\end{aligned}
$$

This implies that $0<\frac{\partial P}{\partial \rho}$ if $P \geq \Delta_{M}$, because

$$
\begin{aligned}
0< & \frac{\partial P}{\partial \rho} \Leftrightarrow 0<\frac{\partial}{\partial \rho} \frac{(1-q) \rho \phi \Delta_{H}+(1-q)(1-\rho) \Delta_{M}+q \Delta_{L}}{1-(1-q) \rho(1-\phi)} \\
\Leftrightarrow & 0<\left[(1-q) \rho \phi \Delta_{H}+(1-q)(1-\rho) \Delta_{M}+q \Delta_{L}\right](1-q)(1-\phi) \\
& +(1-q) \phi \Delta_{H}-(1-q) \rho \phi \Delta_{H}(1-q)(1-\phi)-(1-q) \Delta_{M}+(1-q) \Delta_{M}(1-q) \rho(1-\phi) \\
\Leftrightarrow & 0<\left[(1-q) \Delta_{M}+q \Delta_{L}\right](1-\phi)+\phi \Delta_{H}-\Delta_{M}
\end{aligned}
$$

which holds if $P \geq \Delta_{M}$, because the RHS in (16) is strictly larger than the RHS in (15), that is,

$$
\begin{aligned}
{\left[(1-q) \Delta_{M}+q \Delta_{L}\right](1-\phi)+\phi \Delta_{H}-\Delta_{M} } & >(1-q) \rho \phi \Delta_{H}+q \Delta_{L}-(1-q) \rho \phi \Delta_{M}+(1-q) \Delta_{M}-\Delta_{M} \\
& \Leftrightarrow \Delta_{H}>(1-q) \rho \Delta_{H}+(1-q)(1-\rho) \Delta_{M}+q \Delta_{L},
\end{aligned}
$$


which always holds since $q \geq \underline{q}>0$, where the latter inequality $\underline{q}>0$ follows from (14).

Proof of Proposition 2. First, I show that $V^{*}$ is strictly increasing in $q$ if $q \leq \underline{q}$ and that it attains its unique maximum at $q=\underline{q}$.

The proof of this argument consists of two steps. First, I show that $(1-q) \rho^{*}$ is strictly increasing in $q$ if $q<\underline{q}$. Note that since $P^{*}$ is given by (3) and $P^{*}$ does not change with $q$ if $q<\bar{q}$ by Proposition 1(i), applying Implicit Function Theorem (IFT) on (3) w.r.t. $q$ yields

$$
\begin{aligned}
0= & \frac{d}{d q} \frac{(1-q) \rho^{*}\left[\phi \Delta_{H}-\Delta_{M}\right]+(1-q) \Delta_{M}+q \Delta_{L}}{1-(1-q) \rho^{*}(1-\phi)} \\
0= & {\left[(1-q) \rho^{*}\left[\phi \Delta_{H}-\Delta_{M}\right]+(1-q) \Delta_{M}+q \Delta_{L}\right](1-\phi) \frac{d(1-q) \rho^{*}}{d q} } \\
& +\left\{\frac{d(1-q) \rho^{*}}{d q}\left[\phi \Delta_{H}-\Delta_{M}\right]-\Delta_{M}+\Delta_{L}\right\}\left[1-(1-q) \rho^{*}(1-\phi)\right] \\
\frac{d(1-q) \rho^{*}}{d q}= & \frac{\left(\Delta_{M}-\Delta_{L}\right)\left[1-(1-q) \rho^{*}(1-\phi)\right]}{\phi \Delta_{H}+q(1-\phi) \Delta_{L}-[1-(1-q)(1-\phi)] \Delta_{M}} .
\end{aligned}
$$

Note that $\Delta_{L}<\Delta_{M}$ implies that the numerator of the RHS is positive, therefore it remains to show that the denominator is positive as well. To see this, note that for any $q<\underline{q}$ implies that $\rho^{*}<1$ and hence that $P^{*}>\Delta_{M}$, since if $P^{*} \leq \Delta_{M}$ then (1) would imply that the good agent would strictly prefer to exert effort and receive a payoff of $\phi P^{*}+(1-\phi) \Delta_{H}-\frac{c}{\alpha}$ rather than $\phi P^{*}+(1-\phi) \max \left\{P^{*}, \Delta_{M}\right\}$. Moreover, since Proposition 1 implies that $P^{*}$ is continuous w.r.t. $q$, this also implies that $P^{*} \geq \Delta_{M}$ when $q=\underline{q}$. Since the denominator of the RHS in (18) is strictly decreasing in $q$ (because $\Delta_{L}<\Delta_{M}$ ), it is sufficient to show that it is positive for $q=\underline{q}$. Plugging $P^{*}$ for $q \leq \underline{q}$ from Proposition 1 yields

$\Delta_{M} \leq P^{*} \Leftrightarrow \Delta_{M} \leq \frac{(1-\underline{q}) \phi \Delta_{H}+\underline{q} \Delta_{L}}{1-(1-\underline{q})(1-\phi)} \Leftrightarrow 0 \leq(1-\underline{q}) \phi \Delta_{H}+\underline{q} \Delta_{L}-\Delta_{M}[1-(1-\underline{q})(1-\phi)]$

Since $\underline{q} \in(0,1)$ by Proposition 1 , this implies that the denominator of the RHS in (18) is positive, concluding that $(1-q) \rho^{*}$ is strictly increasing in $q$ if $q<\underline{q}$. Moreover, note that by Proposition $1, \rho^{*}$ and $P^{*}$ are continuous w.r.t. $q$ at $q=\underline{q}$, and hence going through the same 
steps above yields that $(1-q) \rho^{*}$ is also strictly increasing in $q$ from the left at $q=\underline{q}$.

Second, I show that $V^{*}$ is strictly increasing in $q$ if $q<\underline{q}$. Note that (9) and (3) imply that for all $q \in[0,1]$,

$$
V^{*}=\Delta_{H}-\left(\Delta_{H}-P^{*}\right)\left[1-(1-q) \rho^{*}(1-\phi)\right]
$$

Note that $P^{*}<\Delta_{H}$ for all $q \leq \underline{q}$ by Proposition 1. Therefore, since $(1-q) \rho^{*}$ is strictly increasing in $q$ if $q<\underline{q}$ (and also strictly increasing in $q$ from the left at $q=\underline{q}$ ), so is $V^{*}$. This completes the proof of the first argument.

Second, since $\rho^{*}=1$ by Proposition $1,(9)$ and $\Delta_{L}<\Delta_{H}$ imply that $V^{*}$ is strictly decreasing in $q$ in if $q \geq \underline{q}$. Due to the previous step, this implies that $V^{*}$ attains its unique maximum at $q=\underline{q}$.

Finally, I show that $V^{*}(\underline{q})$ strictly increases as $\Delta_{L}$ decreases. Note that by Proposition 1 and the implied continuity of $P^{*}$ w.r.t. $q, P^{*}(\underline{q})$ is given by $(7)$ and does not change w.r.t. $\Delta_{L}$. Since $\rho^{*}=1$ if $q=\underline{q},(19)$ and $P^{*}<\Delta_{H}$ imply that $V^{*}(\underline{q})$ is strictly larger if $\underline{q}$ is smaller. Since

$\underline{q}$ strictly decreases as $\Delta_{L}$ decreases, this implies that $V(\underline{q})$ strictly increases as $\Delta_{L}$ decreases, concluding the proof.

\section{Proof of Lemma 3.}

Note that if there are any value destroying agents in equilibrium that enter, then the agents with good projects strictly prefer to enter. This is because the entry cost of a value destroying agent is $\kappa$, while it is $\kappa-c_{s}$ for the agent with good project. On the other hand, the payoff of the agent with good project after entering is larger than that of value destroying agent.

Therefore, it is sufficient to show that if no value destroying agent enters, then the agent with good project never enters if $\kappa>\hat{\kappa}$ and always enters if $\kappa<\hat{\kappa}$. Note that when no value destroying agents enters, the profit of the agent with good project from entering is $P^{*}(0)-\eta V^{*}(0)-\left(\kappa-c_{s}\right)$, or equivalently, $\hat{\kappa}-\kappa$. This profit includes the entry costs the agent incurs. Therefore, the agent with good project never enters if $\kappa>\hat{\kappa}$ and always enters if $\kappa<\hat{\kappa}$, concluding the proof. 


\section{Proof of Proposition 3.}

Note that $\kappa<\hat{\kappa}$ by assumption, and therefore all of the agents with good projects enter due to Lemma 3.

Suppose that $\kappa>(1-\eta) \Delta_{L}$. First, I prove that $P^{*}(q)-\eta V^{*}(q)$ strictly decreases w.r.t $q$ if $P^{*}(q)-\eta V^{*}(q)>(1-\eta) \Delta_{L}$. Note that by Propositions 1 and $2, P^{*}$ is constant for all $q \in[0, \underline{q}]$, while $V^{*}$ is strictly increasing w.r.t. $q$ for all $q \in[0, \underline{q})$ and is also increasing from the left when $q=\underline{q}$. Therefore, $P^{*}(q)-\eta V^{*}(q)$ strictly decreases w.r.t. $q$ for all $q \in[0, \underline{q})$ and also from the left when $q=q$. Therefore, it remains to show that $P^{*}(q)-\eta V^{*}(q)$ strictly decreases w.r.t $q$ if $q \geq \underline{q}$ as well. Since then Proposition 1 implies that $\rho^{*}=1$, and hence

$$
P-\eta V=\frac{\phi \Delta_{H}+q\left[\Delta_{L}-\phi \Delta_{H}\right]}{1-(1-q)(1-\phi)}-\eta\left\{\Delta_{H}+q\left[\Delta_{L}-\Delta_{H}\right]\right\}
$$

Therefore,

$$
\begin{aligned}
0 & >\frac{d(P-\eta V)}{d q} \Leftrightarrow 0>\frac{\left[\Delta_{L}-\phi \Delta_{H}\right]}{1-(1-q)(1-\phi)}-\frac{\phi \Delta_{H}+q\left[\Delta_{L}-\phi \Delta_{H}\right]}{[1-(1-q)(1-\phi)]^{2}}(1-\phi)-\eta\left\{\left[\Delta_{L}-\Delta_{H}\right]\right\} \\
& \Leftrightarrow \frac{\phi \Delta_{H}+q\left[\Delta_{L}-\phi \Delta_{H}\right]}{[1-(1-q)(1-\phi)]^{2}}(1-\phi)+\eta\left\{\left[\Delta_{L}-\Delta_{H}\right]\right\}>\frac{\left[\Delta_{L}-\phi \Delta_{H}\right]}{1-(1-q)(1-\phi)} \\
& \Leftrightarrow\left[\phi \Delta_{H}+q\left[\Delta_{L}-\phi \Delta_{H}\right]\right](1-\phi)+\eta\left\{\left[\Delta_{L}-\Delta_{H}\right]\right\}[1-(1-q)(1-\phi)]^{2} \\
& >\left[\Delta_{L}-\phi \Delta_{H}\right](1-(1-q)(1-\phi)) \\
& \Leftrightarrow \eta\left\{\left[\Delta_{L}-\Delta_{H}\right]\right\}[1-(1-q)(1-\phi)]^{2}>\phi\left[\Delta_{L}-\Delta_{H}\right] \Leftrightarrow \eta[1-(1-q)(1-\phi)]^{2}<\phi \\
& \Leftrightarrow q<q^{\prime} \equiv 1-\frac{1-\sqrt{\phi / \eta}}{1-\phi}
\end{aligned}
$$

where the RHS is strictly larger than 0 . Moreover, since $P^{*}(q)=V^{*}(q)=\Delta_{L}$ and hence $P^{*}(q)-\eta V^{*}(q)=(1-\eta) \Delta_{L}$ if $q=1$, this implies that $P^{*}(q)-\eta V^{*}(q)$ strictly decreases w.r.t $q$ if $P^{*}(q)-\eta V^{*}(q)>(1-\eta) \Delta_{L}$ and $q \geq \underline{q}$, concluding the proof of this step.

Second, I derive (11). There are two cases to consider. Suppose that $\kappa \geq P^{*}(0)-\eta V^{*}(0)$. Since $\kappa>(1-\eta) \Delta_{L}$ is also satisfied by assumption, by the first step above this implies that $\kappa>P^{*}(q)-\eta V^{*}(q)$ for all $q \in(0,1]$, and therefore the equilibrium is unique and given by 
$q^{*}=0$. Next, suppose that $\kappa<P^{*}(0)-\eta V^{*}(0)$. Then, it can never be $q=0$ or $q=1$ in equilibrium, since in the former the agent with bad project strictly prefers to enter, and in the latter the agent with bad project strictly prefers to exit since his gross profit from entering satisfies $P^{*}(q)-\eta V^{*}(q)=(1-\eta) \Delta_{L}<\kappa$. Therefore, in equilibrium it must be that $\kappa=P^{*}(q)-\eta V^{*}(q)$. Since $\kappa>(1-\eta) \Delta_{L}$ by assumption and $P^{*}(q)-\eta V^{*}(q)$ strictly decreases w.r.t $q$ if $P^{*}(q)-\eta V^{*}(q)>(1-\eta) \Delta_{L}$, the continuity of $P^{*}(q)-\eta V^{*}(q)$ implies that there is a unique $q \in(0,1)$ that satisfies $\kappa=P^{*}(q)-\eta V^{*}(q)$, concluding the proof.

Proof of Proposition 4. Note that $\kappa<\hat{\kappa}$ by assumption, and therefore all of the agents with good projects enter due to Lemma 3. Suppose that $\Delta_{L}<0$ and $\kappa \geq 0$. Then, the equilibrium is unique by Proposition 3. Moreover, also recall that by Proposition $3, P^{*}(q)-\eta V^{*}(q)$ strictly decreases w.r.t $q$ if $P^{*}(q)-\eta V^{*}(q)>(1-\eta) \Delta_{L}$, which I will use throughout the proof.

Consider part (i), and suppose that $\eta \geq P^{*}(0) / V^{*}(0)$. Then, $P^{*}(0)-\eta V^{*}(0) \leq 0$, and since $(1-\eta) \Delta_{L}<0$ as well, Proposition 3 implies that $P^{*}(q)-\eta V^{*}(q)<0$ for all $q \in(0,1]$. Therefore, $\kappa \geq 0$ implies that the equilibrium must satisfy $q^{*}=0$.

Consider part (ii), and suppose that $\eta<P^{*}(0) / V^{*}(0)$. Note that then $\bar{\kappa} \equiv P^{*}(0)-$ $\eta V^{*}(0)>0>(1-\eta) \Delta_{L}$. In turn, by Proposition 3, this implies that $P^{*}(\underline{q})-\eta V^{*}(\underline{q})<$ $P^{*}(0)-\eta V^{*}(0)$. Therefore, $P^{*}(\underline{q})-\eta V^{*}(\underline{q})<\bar{\kappa}$, resulting in $0 \leq \underline{\kappa}<\bar{\kappa}$.

Consider part (ii.a), and suppose that $\kappa>\bar{\kappa}$. Then, Proposition 3 implies that $q^{*}=0$, and hence $V^{*}$ does not change w.r.t. $\kappa$.

Consider part (ii.b), and suppose that $\kappa \in(\underline{\kappa}, \bar{\kappa}]$. Then, by Proposition 3, the equilibrium satisfies $P\left(q^{*}\right)-\eta V\left(q^{*}\right)=\kappa$, and since $\kappa>(1-\eta) \Delta_{L}, P(q)-\eta V(q)$ strictly decreases w.r.t. $q$ at $q=q^{*}$. Therefore, as $\kappa$ decreases, $q^{*}$ increases. In turn, Proposition 2 implies that $V\left(q^{*}\right)$ strictly increases.

Consider part (ii.c), and suppose that $\kappa \in[0, \underline{\kappa}]$. Then, $P^{*}(\underline{q})-\eta V^{*}(\underline{q}) \geq 0$, and $\kappa \geq 0>$ $(1-\eta) \Delta_{L}$. Then, by Proposition 3, again the equilibrium satisfies $P\left(q^{*}\right)-\eta V\left(q^{*}\right)=\kappa$, and $P(q)-\eta V(q)$ strictly decreases w.r.t. $q$ at $q=q^{*}$. Therefore, $q^{*}$ increases as $\kappa$ decreases. In turn, Proposition 2 implies that $V\left(q^{*}\right)$ strictly decreases. 
Finally, in all of the above cases, note that the continuity of $P^{*}(q)-\eta V^{*}(q)$ w.r.t. $q$ implies that $q^{*}$ and $V^{*}$ are continuous w.r.t. $\kappa$ as well, and hence $V^{*}$ attains its global maximum w.r.t. $\kappa \in[0, \infty)$ at $\kappa=\underline{\kappa}$. 